\title{
In search of the sample: Recent experiences of a trial team in Orthodontics
}

\author{
Susan Cunningham ${ }^{\mathrm{a}, *}$, David Bearn $^{\mathrm{b}}$, Philip Benson ${ }^{\mathrm{c}}$, Ama Johal ${ }^{\mathrm{d}}$, Declan Millett ${ }^{\mathrm{e}}$, \\ Kevin O'Brien ${ }^{\mathrm{f}}$, Friedy Luther ${ }^{\mathrm{c}}$ \\ a Department of Orthodontics, UCL Eastman Dental Institute, London, United Kingdom \\ ${ }^{\mathrm{b}}$ Department of Orthodontics, University of Dundee, United Kingdom \\ ${ }^{c}$ Department of Orthodontics, University of Sheffield, United Kingdom \\ d Department of Orthodontics, St Bartholomew's and The London Queen Mary's School of Medicine and Dentistry, United Kingdom \\ e Department of Orthodontics, University College Cork, Ireland \\ ${ }^{\mathrm{f}}$ Department of Orthodontics, University of Manchester, United Kingdom
}

\section{A R T I C L E I N F O}

\section{Article history:}

Received 28 October 2010

Accepted 5 April 2011

Available online 17 April 2011

\section{Keywords:}

Randomised clinical trial

Recruitment difficulties

Orthodontics

\begin{abstract}
A B S T R A C T
Randomised clinical trials are commonly undertaken in medical and dental research. However, few authors discuss the difficulties associated with such studies, including costs, ethical issues and recruitment of an adequate number of patients. There is little information available on the number of studies which are terminated early as a result of these issues, but it seems likely that at least $10 \%$ of clinical studies never reach completion.

This paper reviews two nationally funded clinical studies which were terminated early. It highlights the problems associated with patient recruitment and also some issues which may benefit researchers undertaking similar studies in the future.
\end{abstract}

(c) 2011 Elsevier Inc. All rights reserved.

\section{Introduction}

The emphasis on the 'hierarchy of evidence' is increasing, with randomised clinical trials recommended as one of the best sources of scientific evidence [1]. However, there is less discussion of the difficulties associated with undertaking such studies, including costs [2], ethical problems, informed consent [3], problems of bias, clinician preference for certain treatment options [4], difficulties in recruiting sufficient patients [5] and overestimating the number of patients who would be eligible for inclusion in the study or would agree to participate [6].

It is, therefore, likely that some clinical trials are not completed, although there is little information in the literature regarding those studies and the reasons for this. The problem is compounded by the fact that many studies fail to reach an adequate sample size, but it appears that they are rarely registered as failing to complete. For example, one

\footnotetext{
* Corresponding author at: Department of Orthodontics, UCL Eastman Dental Institute, 256 Grays Inn Road, London, WC1X 8LD, United Kingdom. Tel.: + 442079151064 .

E-mail address: S.Cunningham@eastman.ucl.ac.uk (S. Cunningham).
}

London teaching hospital estimated that between 5 and 10\% of clinical studies approved by their Ethics Committee each year were terminated early and completed the necessary paperwork (Personal Communication, 2009).

If the figures are of this magnitude then it is important that we, as researchers, consider carefully why studies are not completed and how this can guide future research planning. This paper reviews two nationally funded clinical studies in the field of Orthodontics, a sub-specialty of Dentistry devoted to managing developmental anomalies of the jaws and teeth, which were terminated early. The aim is to highlight the under-reported problems associated with patient recruitment and to highlight some issues which may benefit researchers undertaking similar studies in the future.

\section{Study 1}

\subsection{Introduction}

This multi-centre study compared two different methods of treating a specific type of malocclusion (a problem with the way the teeth bite together) in adolescents. The research 
question was: Is there any difference between treatment with a fixed brace and removal of two teeth from the upper jaw compared with treatment with a removable brace followed by a fixed brace but avoiding the need for extractions with respect to:

\section{- Treatment duration}

- Number of visits

- Patient discomfort

- Comparison of pre- and post-treatment radiographs of the teeth and jaws

- Comparison of pre- and post-treatment study casts of the teeth and jaws

- Jaw joint symptoms.

The protocol was designed within a multi-centre health services research group comprising senior clinical academics in the UK, all with higher research degrees (PhD or DDS) and each with a minimum of 10 years research experience. Nine operators (working in six hospital departments) participated in the study, including one academic who had previously run a large multi-centre study funded by the UK Medical Research Council.

The sample size calculation used the difference in treatment duration as the primary outcome measure, with a clinically meaningful difference of 6 months [7]. Forty patients were required in each of the two groups and, as the drop-out rate from similar investigations was around $20 \%$, it was decided to recruit 50 patients in each arm of the study. Within the UK population, the prevalence of the particular malocclusion which was being investigated is reported to be $10 \%$ [8] and it was anticipated that this would be reflected in the patients being referred to new patient clinics within the hospital service in the UK.

Funding and multi-centre ethical approval were obtained. Following an orientation day in late 2005, recruitment commenced in mid-2006.

\subsection{Difficulties encountered}

At the planned 6 month trial team meeting, it became clear that recruitment was a problem and a number of the researchers reported that they had seen a relatively low number of patients with this type of dental problem in their own hospitals. Furthermore, some of those they had seen did not fulfil the inclusion criteria and therefore could not be recruited. Therefore all researchers returned to their units to assess waiting lists and to contact local general dental practitioners asking them to refer suitable patients. It was also reported that a small number of patients who were eligible had refused to participate in the trial as they had a clear view of which treatment they wished to undergo. Interestingly, there were patients allocated to both intervention groups who wished to undergo the other form of treatment, hence it was not that one option was obviously preferred to the other. It was therefore decided to introduce a 'preference group' in an attempt to improve recruitment, whilst acknowledging the effect this would have on sample size. This amendment was approved by the ethics committee.

By April 2007, only 16 patients had been recruited and the trial team met in July 2007 to review the situation. It was agreed that, although the study had been designed by an experienced research team with a robust protocol, the number of patients being referred was clearly not as high as had been anticipated. The team reluctantly accepted that it was highly unlikely that 100 patients could be recruited within a reasonable timeframe and terminated the trial. All patients who had been recruited were informed of the decision to stop the trial, were thanked for their help and assured that their care would continue exactly as before.

\subsection{Comment}

The treatment options compared in this study had not been studied in detail before nor had any RCTs been undertaken. In hindsight, maybe this should have heightened the researchers' awareness of the potential difficulties of recruitment. It became apparent early in the study that the research team had over-estimated the number of patients with this type of malocclusion being referred to the hospital service and had underestimated how long it would take to recruit the 100 patients needed. This may have been due to the prevalence data [8] being historic or the prevalence was correct but the malocclusions were not sufficiently severe for the individual to be referred.

The team initially had some concerns regarding patients' perceptions of RCTs and whether this may have influenced recruitment, hence the decision to introduce a preference group. However, recruitment was not resolved by the introduction of this option so this did not appear to be the major issue.

All six of the participating hospitals had recruited to the study. Unfortunately, due to the very small number of patients recruited, it was not possible to establish whether any hospital was more successful than the others. Nevertheless, this may be an important issue to consider in RCT recruitment if such problems are encountered.

\section{Study 2}

\subsection{Introduction}

This was a single centre, prospective clinical study using magnetic resonance imaging (MRI) to investigate if an association exists between jaw joint/temporomandibular joint (TMJ) status and clinical parameters pertaining to malocclusion. The protocol was designed within a singlecentre health services research team comprising two senior clinical academics (both with $\mathrm{PhD}$ qualifications and each with over 10 years of research experience), one NHS consultant in radiology and one lecturer in biostatistics.

A preliminary survey of 100 consecutive new patients in the Orthodontic Department indicated that $75 \%$ of patients would be willing to attend voluntarily on a Sunday for an MRI scan (Sunday was "down-time" for the MRI unit and therefore caused least interference to normal activities). A grant was obtained to allow funding of the scans and to allow patients to be given a $£ 15$ voucher for participating. Following ethical approval, recruitment commenced in October 2004. New patients who met the inclusion criteria and were willing to take part in the study underwent a routine clinical dental assessment and, following this, an MRI scan. 
The data to be collected possessed a hierarchical structure, thus multilevel modelling statistical techniques were to be applied to the data. It was assumed that if a full set of observations was made, each 'subject' would have two joints for investigation and each right and left joint had two associated 'views' (coronal and sagittal), with six associated 'condition' measurements. A sample size calculation taking this hierarchical structure into account estimated that 320 subjects were required.

\subsection{Difficulties encountered}

Recruitment proved difficult from the outset. The inclusion criteria were relatively straightforward: patients had to be at least 10 years old, but with no previous dental extractions or orthodontic ("brace") therapy. Recruiting clinicians reported that approximately $30 \%$ of new patients were adults and that many had undergone previous extractions and were therefore excluded at the outset. In addition, unforeseen changes in staffing resulted in the loss, or reduced participation, of four recruiting clinicians. Clinicians who were involved in recruitment also acknowledged that they frequently forgot to invite suitable patients, due to time pressures on clinics.

Steps taken to address these problems included regular reminders to recruiting staff; attempts to engage new staff in the study; and the main researcher visiting new patient clinics to recruit, in order to reduce the load on the clinician running that clinic. At this stage, consideration was given to making the study a multi-centre investigation but cost implications suggested that this was not feasible. The study had only been possible on the main site due to negotiations by the consultant radiologist to reduce MRI costs for this project.

By October 2005, 60 patients had been invited to participate but only 13 had consented. Negotiation within the department allowed access to a research nurse to recruit patients for a limited number of sessions and by October 2006, 102 patients had been invited but only 21 patients had consented. An extension to the grant was applied for, whilst protocol/ethical amendments were made to extend the research to include healthy volunteers. During October 2006, the study was advertised throughout the university campus and initially this appeared successful with over 100 enquiries. However, only 40 volunteers followed up their enquiry, and 20 met the inclusion criteria and were recruited.

Ultimately, 38 patients and volunteers were recruited but the total number of patients/volunteers actually scanned was just 19, reflecting a high failure rate for attendance for the scans. As a result of ongoing recruitment problems, the research team and funders decided to terminate the study.

\subsection{Comment}

The orthodontic literature gave no suggestion of recruitment problems associated with MRI scanning. However, previous studies generally only involved about 100 patients (sometimes fewer) and were not based on sample size calculations. Consequently, the research team was always aware that the project would rely on the willingness of patients (and later also volunteers) to undergo a non-routine procedure of no immediate benefit to them involving an extra appointment. The high failure rate for the scan itself suggested that volunteers were more willing to have a dental assessment than attend for the scan. Furthermore, the number of patients who had to be excluded was higher than expected.

Access to the research nurse was a considerable help and had the nurse been available initially and for all new patient clinics the situation may have improved, although this alone would not have resolved all of the difficulties.

\section{General discussion}

Both studies reported were terminated due to recruitment problems but an analysis of factors they had in common, and factors that were not, is instructive.

Factors in common between the studies:

- Both were clinical studies involving experienced academics, therefore experience of clinical research per se was not an issue.

- Both were based on power calculations which almost certainly resulted in larger sample sizes than the convenience samples or retrospective samples much of the previous research is based on.

- Both were reliant on having a good source of patients. However, the estimated recruitment period was based on what would appear to be inaccurate data regarding the prevalence of the condition (although this was the best available data at the time) [Study 1] or patient willingness to attend for scans [Study 2].

\section{Specific factors affecting the studies:}

- Study 1 was a multi-centre RCT, whilst study 2 was a singlecentre study yet neither succeeded.

- Study 1 involved finding suitable patients, but when eligible patients were invited to take part some were not willing to do so. In contrast, Study 2 ultimately involved patients and volunteers. The patients again had to be found, but the percentage of patients agreeing to take part was lower than for Study 1. The volunteers seemed willing to take part in the clinical part of the study but many failed to attend for the scan.

- Study 2 was able to include a research nurse later in the study aiding recruitment, but this could not resolve all of the significant issues in either study.

Data on failure rates of clinical studies and the causes have proved hard to obtain. Arshad and Arkwright [9] undertook a prospective questionnaire-based survey sent to all principal investigators who submitted ethics applications to nine Greater Manchester Research Ethics Committees (RECs) between April 2004 and March 2005. Data on the outcome and status of the REC applications and studies were analysed. Of the 506 questionnaires sent out, only 288 (57\%) were returned. Based on this response rate, $97 \%$ of REC applications were approved (although data from the RECs themselves indicated $9 \%$ were rejected) and $87 \%$ of studies were in progress or had been completed 1 to 2 years after approval had been granted. This indicates that whilst the ethics approval process can be onerous in the UK, most studies do gain approval and proceed. However, given the response rate, 
the authors acknowledge that their data may be biassed and under-report failures: of those studies approved and started, $4 \%$ had to be abandoned or postponed. The commonest reasons were an inability to recruit patients and/or loss of research staff.

Data supplied by a second NHS Research and Development (R\&D) Department in the UK indicated that, of a total of 840 active studies, 91 had been withdrawn, suggesting a "failure rate" of $10.8 \%$. The reasons for withdrawal were generally not provided to the R\&D Department.

It is a concern that 1 in 10 clinical studies is likely to fail and the most likely reasons appear to be recruitment and/or staffing problems. Many examples in the literature demonstrate that recruitment is a problem affecting medical research but no reports appear to exist for dentistry/ orthodontics. Studies in all of the following have experienced problems: primary care [10]; HIV clinical trials [11]; asthma [12]; psychiatric studies [13] and oncology [14].

Lovato et al. [11] discussed many issues hindering recruitment, including barriers to recruitment of diverse populations due to language and cultural factors, beliefs about medical research, and the appropriateness of available protocols. Furthermore, recruitment strategies such as patient registries, occupational screening, direct mail, and the media have been prominent in the literature since at least 1997. They suggest that successful planning and management should include piloting strategies, monitoring recruitment by data tracking systems, and hiring quality staff.

However, not all of these suggestions are useful in a dental situation and certain factors make clinical research in the field of orthodontics particularly difficult, including: recruiting children and requiring consent from parents/guardians; the long term nature of the treatment and follow-up (treatment usually takes in the region of 2 years from start to finish); and problems associated with different clinicians undertaking different treatment planning and mechanics. Even socioeconomic status may be a factor affecting recruitment [15] and may represent a further hurdle which needs to be addressed.

Recruitment issues clearly remain a significant problem associated with undertaking clinical trials [16]. Various means are being used to address these problems and often stem from pharmaceutical companies who may lose vast sums when recruitment is delayed $[17,18]$. Clinical trials are increasingly being undertaken outside of the US and Western Europe. For example, one paper [19] reported that in 2008 in the USA, $80 \%$ of approved marketing applications for drugs and biologics contained data from foreign clinical trials. Over half of clinical trial subjects and sites were located outside the United States; Western Europe accounted for most foreign clinical trial subjects and sites, however, Central and South America had the highest average number of subjects per site.

In the USA, consideration is now being given to the use of social networks, such as Facebook and Twitter, to advertise the running of clinical trials [18]. Just as patient information leaflets would be sent to the ethics body for approval prior to use, the same applies to the use of social networks for advertising a clinical trial and recruiting to it. Therefore there seems to be no reason in principle why this method should not be used elsewhere. Whether these new methods actually lead to increased recruitment is still open to debate, since they are only just starting to be used [18]. Murphy and
Merenstein [20] reported success in a Paediatric trial in which they compared so-called grassroots campaigning with traditional methods. The grassroots approach involved identifying subjects through, for example, community and day care centres, churches and local websites. Staff spent time in the community, thus allowing better contact with participants, better follow-up rates etc. Traditional techniques included the more usual approach of recruiting at clinics and hospitals by identifying those patients already receiving treatment and then contacting them to invite participation. They did, however, highlight the problems of obtaining research funding to be used in this way.

Furthermore, whilst potentially new recruitment methods could be used anywhere in the world, their effectiveness and feasibility are likely to be limited by simple practical differences between the developing and developed world. Tornieporth [21] makes the point that whilst more and more countries are organising and developing their ethical review boards, huge differences exist between countries and continents and this issue is yet to be addressed. Similarly, one would imagine that simple lack of access to computers and the internet would be the main factor stopping some populations from being accessed via these new "high tech" means.

For study 1, such widespread and relatively broad brush advertising would not have been appropriate as, in this case, a specific form of malocclusion was being sought. Whilst patients frequently realise that their teeth are not straight, the subtleties beyond that require training to recognise the type of malocclusion. Furthermore, those patients eligible for treatment within the National Health Service are restricted by the UK Department of Health and advertising in this case may have resulted in inappropriate individuals coming forward and resources being wasted in simply screening those individuals out again.

For study 2, it would have been possible to "advertise" more widely, i.e. beyond the university campus, but both funding and personnel resources were limited. Therefore, only free advertising methods were considered and, since there were only two people available (part-time and for limited periods) to screen individuals for the study, so the level of advertising was limited by both of these issues. If the study were to be undertaken now, the use of social networks could be considered but, at the time, these were only just becoming popular.

Recruitment to clinical trials may also be influenced by the health care system in that country. In the UK, where these trials were undertaken, the treatment would be paid for whether the subject participated in the trial or not so there is little incentive for the subject to participate. On the other hand, in a country with only private pay options, there would be greater incentive for the subject to participate if the treatment was paid for by the study. In Study 2, volunteers were given a voucher as a thank you for taking part. The question as to whether such gifts constitute coercion or an honorarium has been much debated and the question which must be asked is "Do financial incentives affect behaviour and cause volunteers to act against their normal judgment?". Groth [22] studied the evidence on this and concluded that financial recompense was a way of increasing recruitment but volunteers took multiple factors into account. Groth [22] discussed a number of these issues and concluded that costs, such as for travel to a research site, were a 
factor when individuals decide whether or not to become involved in a research study. This is considered alongside the personal value of participation and when this personal value exceeds the aggravation and costs that go along with involvement, then the patient may consider taking part. It was felt that those studies which did not incur participant costs had fewer recruitment problems but financial incentives may still be a way of compensating the participant and acknowledging their time and commitment. It may be that had the vouchers in Study 2 been of a larger value, this would have improved recruitment; however the financial constraints prevented this.

The final question is "Where do we go from here?". Clearly, research should be planned by experienced researchers who have a full understanding of the associated problems to reduce the possibility of study termination becoming necessary, but this alone is not sufficient as the paper clearly illustrates. The dental and orthodontic elements of this research have raised some specific factors but there are also general issues which researchers may find useful to consider in the future. Feasibility studies to determine likely participation rates would appear to be a particularly useful first step as these may alert researchers to potential difficulties, especially when planning is otherwise based on historic data or data from previous studies where the methodology may be questionable. The employment of research nurses undoubtedly aids recruitment in clinical studies. The corollary is that the costs of running studies will increase and this is something which grant awarding bodies must acknowledge if good quality clinical research is to continue.

The problems that occurred with these two studies provide some weight to the argument that feasibility studies should be carried out before applications are made for funding. These would provide information on the selection of outcomes, number of eligible subjects attending for care with the condition of interest, the likely recruitment rate, willingness of clinicians to randomise and willingness of patients to be randomised. This approach has been adopted in trials of recurrent cellulites of the leg [23] and cystic fibrosis [24]. Furthermore, it has also been suggested that there should be an economic assessment of the value of carrying out additional research relative to the cost of further research by carrying out a Value of Information Analysis. This approach has been suggested in a position paper to the Health Technology Assessment programme of the National Institute for Health Research and this could certainly be of use in the allocation of scarce resource to help identify those studies that are most likely to complete and provide useful clinical information [25].

\section{References}

[1] Manchikanti L, Hirsch JA, Smith HS. Evidence-based medicine, systematic reviews, and guidelines in interventional pain management: part 2: randomized controlled trials. Pain Phys 2008;11:717-73.

[2] Malakoff D. Clinical trials and tribulations. Spiraling costs threaten gridlock. Science 2008;322:210-3.

[3] Juraskova I, Butow P, Lopez A, Seccombe M, Coates A, Boyle F, et al. Improving informed consent: pilot of a decision aid for women invited to participate in a breast cancer prevention trial (IBIS-II DCIS). Health Expect 2008;11:252-62.

[4] Walter SD, Ismaila AS, for the SPRINT Study Investigators. Statistical issues in the design and analysis of expertise-based randomized clinical trials. Stat Med 2008;27:6583-96.

[5] Pethig K, Hornig B, Bara C, Schieffer B, Haverich A, Sachse A. Eprosartan in the primary prevention of cardiac allograft vascular disease: a double-blind prospectively randomized study using intravascular ultrasound. J Int Med Res 2008;36:1022-31.

[6] Jolly K, Tayor RS, Lip GY, Greenfield SM, Davies MK, Davis RC, et al. Home-based exercise rehabilitation in addition to specialist heart failure nurse care: design, rationale and recruitment to the Birmingham Rehabilitation Uptake Maximisation study for patients with congestive heart failure (BRUM-CHF): a randomised controlled trial. BMC Cardiovasc Disord 2007;7:9.

[7] O'Brien K, Wright J, Conboy F, Sanjie Y, Mandall N, Chadwick S, et al. Effectiveness of treatment for Class II malocclusion with the Herbst or twin-block appliances: a randomized, controlled trial. Am J Orthod Dentofacial Orthop 2003;124:128-37.

[8] Houston WJB, Stephens CD. Tulley WJ A textbook of orthodontics. Butterworth-Heinemann Ltd.; 1996.

[9] Arshad A, Arkwright PD. Status of healthcare studies submitted to UK research ethics committees for approval in 2004-5. J Med Ethics 2008;34:393-5.

[10] Hoddinott P, Britten J, Harrild K, Godden DJ. Recruitment issues when primary care population clusters are used in randomised controlled clinical trials: climbing mountains or pushing boulders uphill? Control Clin Trials 2007;28:232-41.

[11] Lovato LC, Hill K, Hertert S, Hunninghake DB, Probstfield JL. Recruitment for controlled clinical trials: literature summary and annotated bibliography. Control Clin Trials 1997;18:328-52.

[12] Sorkness CA, Ford JG, Lemanske Jr RF. (Asthma Clinical Trials Network) Recruitment strategies in the Asthma Clinical Research Network. Control Clin Trials 2001;22(6 Suppl):222S-35S

[13] Hinshaw SP, Hoagwood K, Jensen PS, Kratochvil C, Bickman L, Clarke G, et al. AACAP 2001 research forum: challenges and recommendations regarding recruitment and retention of participants in research investigations. J Am Acad Child Adolesc Psychiatry 2004;43:1037-45.

[14] Street A, Strong J, Karp S. Improving patient recruitment to multicentre clinical trails: the case for employing a data manager in a district general hospital-based oncology centre. Clin Oncol (R Coll Radiol) 2001;13:38-43.

[15] Tatsi C, Luther F, Toumba KJ, Duggal M. Socio-economic status-might it affect clinical trial recruitment? J Dent Res 2010;89(Special Issue B) abstract number: 4629. www.dentalresearch.org.

[16] Ganguli P. Getting to know you. Clinical Discovery. http://www.clinical discovery.com/readArticle.aspx?articleId=95, 2011 (accessed 7-3-2011).

[17] Business Insights Report published by. Patient Recruitment and Retention in Clinical Trials: Emerging strategies in Europe the US and Asia. http://www.reportbuyer.com/business_government/advertising marketing/patient_recruitment_retention_clinical_trials.html, June 2007 (accessed 7-3-2011).

[18] Pharma Strategy Blog. The increasing use of social media to recruit patients for clinical trials (posted November 17, 2009), Maverick NY. http://pharmastrategyblog.com/2009/11/the-increasing-use-of-socialmedia-to-recruit-patients-for-clinical-trials.html/ (accessed 7-3-11).

[19] Greenleaf JM, Hereford RW. Challenges to FDA's ability to monitor and inspect foreign. Clinical trials Department of Health and Human Services, Office Of Inspector General; June 2010 http://webcache.googleusercontent. $\mathrm{com} /$ search?oe $=$ utf-8\&rls = org.mozilla\%3Aen-US\%3Aofficial\&client= firefox-a\&hl=en\&q= cache:ySWnPWHRZHoJ:http://oig.hhs.gov/oei/ reports/oei-01-08-00510.pdf + Percentage + of + clinical + trials + taking + place + outside + the + USA + and + Europe $\& \mathrm{ct}=\mathrm{clnk}$ (Accessed 7-3-11).

[20] Murphy M, Merenstein D. Grassroots campaign trail methods to recruit for clinical trials: recruitment lessons learned from trail to trial. Clin Med Insights Pediatr 2011;5:1-7.

[21] Tornieporth N. Ethical aspects of conducting clinical trials in developing countries: Experiences of GlaxoSmithKline (GSK): In: The ethical aspects of biomedical research in developing countries. Proceedings of the Round Table Debate, Brussels; October 1st 2002. p. 53-8.

[22] Groth SA. Honorarium or coercion: use of incentives for participants in clinical research. Journal of the New York State Nurses Association; 2010. Available at: http://www.thefreelibrary.com/Honorarium+or+coercion\% $3 \mathrm{~A}+$ use + of + incentives + for + participants + in ...-a0242591405 (accessed 7-3-11)

[23] Thomas K, Cox N, Savelyich B, Shipley D, Meredith S, Nunn A, et al. Feasibility study to inform the design of a UK multi-centre randomised controlled trial of prophylactic antibiotics for the prevention of recurrent cellulitis of the leg. Trials 2007;8(1):3.

[24] Hickey HR, Jones AP, Lenney W, Williamson PR, Smyth RL. Feasibility study to inform the design of a randomised controlled trial to eradicate Pseudomonas aeruginosa infection in individuals with cystic fibrosis. Trials 2010;11:11.

[25] Claxton K, Ginnelly L, Sculpher M, Philips Z, Palmer S. A pilot study on the use of decision theory and value of information analysis as part of the NHS Health Technology Assessment programme. Health Technol Assess 2004;8(31). 